\title{
Predictors of mortality in rhinocerebral mycosis
}

\author{
Sita S. Jayalakshmi, Ramana G. Reddy, Rupam Borgohain, C. Subramanyam*, Manas Panigrahi ${ }^{\dagger}$, \\ C. Sundaram $¥$, A. K. Meena, S. Mohandas \\ Departments of Neurology, ${ }^{\dagger}$ Neurosurgery, ${ }^{\ddagger}$ Pathology, Nizam’s Institute of Medical Sciences, Punjagutta, Hyderabad - $500082,{ }^{\star}$ Govt. ENT \\ Hospital, Koti, Hyderabad, India
}

\begin{abstract}
Introduction: Rhinocerebral mycosis is a rapidly progressive fatal opportunistic infection, predominantly affecting people in an immunocompromised state. Aggressive surgical therapy, with repeated debridement in combination with intravenous amphotericin B can lead to a high rate of cure. Aim: To determine the predictors of mortality in rhinocerebral mycosis. Materials and Methods: The demographic data, clinical features, radiological (MRI/CT) findings, treatment details of patients with a diagnosis of rhinocerebral mycosis confirmed on histopathology were analyzed retrospectively. The outcome was assessed as alive and dead. Univariate analysis with odds ratio $(\mathrm{OR})$ was employed in data analysis. Chi-square test was used for $P$ value. Results: There were 38 patients. The age range was 7-82 (mean 48.68) years; 30 $(79 \%)$ were males. Craniofacial pain was the most common initial presenting symptom, noted in $29(76.3 \%)$. Rhino-orbital involvement was noted in $24(63.2 \%)$ and $12(31.6 \%)$ had associated focal neurological deficits. Immunocompromised state was noted in $24(63.2 \%)$. Eighteen $(47.4 \%)$ patients died. The predictors for mortality: odds ratio $(95 \% \mathrm{Cl})$ were 2.45 (1.01-3.89) for elderly age, 5.67 (4.13-7.21) for intracranial extension, 2.6 (1.26-3.94) for immunocompromised state, 2.62 (1.25-3.99) for infection with zygomycosis and 2.33 (1.01-3.65) for anemia. Conclusion: Rhinocerebral mycosis is associated with high mortality in spite of aggressive therapy. Intracranial extension with focal neurological deficits is a major predictor of mortality in rhinocerebral mycosis.
\end{abstract}

Key words: Intracranial extension, mortality, rhinocerebral mycosis

Paltauf ${ }^{[1]}$ first identified rhinocerebral mycosis in 1885. He described a case of rhinocerebral mucormycosis. Aspergillosis and zygomycosis are the commonest causes of central nervous system (CNS) mycosis. Cryptococcus, Candida, coccidioides, histoplasma and cladosporium are the other fungi causing CNS mycoses. Aspergillosis is caused by Aspergillus fumigatus in the majority of cases, but A. flavus, A. glaucus and A. niger are not rare.
Zygomycosis includes the fungi of family Mucoraceae. It includes Mucor, Rhizopus, Rhizomucor and Absidia. ${ }^{[2]}$

In rhinocerebral mycosis the disease originates in the nasal/sinus mucosae, after inhalation of fungal spores and takes a rapidly progressive course by extending to neighboring tissues including the orbit and central nervous system. ${ }^{[3]}$ The initial clinical findings in orbital involvement are most commonly headache, lethargy and facial pain. These may be rapidly followed by restriction of ocular movements, proptosis, ptosis and periorbital cellulitis. Paralysis of the third, fourth and sixth cranial nerves and visual loss due to central retinal artery occlusion is not uncommon. The fifth and seventh cranial nerves may be involved. The most serious complication of rhinocerebral mycosis is cerebral vasculitis due to direct vascular involvement. ${ }^{[4]}$

Rhinocerebral mycosis is a rapidly progressive and fatal opportunistic infection, predominantly affecting individuals in an immunocompromised state. Occurrence in immunocompetent individuals is not uncommon. ${ }^{[5]}$ It carries a high residual morbidity and mortality due to the angioinvasive property of fungi, causing vascular occlusion and extensive tissue necrosis. Impaired delivery of the antifungal drugs to the site of infection because of vascular thrombosis and limited aggressive surgery because of the complex anatomy of the rhinoorbitocerebral region cautions for early diagnosis and aggressive management in these patients.

There are many case reports / series of rhinocerebral mycosis. The mortality in rhinocerebral mycosis ranged from $15-82 \%$ in various series. ${ }^{[6-16]}$ The predictors of poor outcome were intracranial extension of the disease, ${ }^{[7,12]}$ delayed diagnosis ${ }^{[9]}$ and hemiplegia as a neurological complication. ${ }^{[11]}$ Aggressive surgical therapy, with repeated debridement in combination with intravenous amphotericin B can lead to high rate of cure with longterm survival rate. ${ }^{[10,15]}$

Part of the work was presented at the $14^{\text {th }}$ annual meeting of the Indian Academy of Neurology, $6-8^{\text {th }}$ October at Bangalore 


\section{Aim}

To determine the predictors of mortality in patients with rhinocerebral mycosis.

\section{Materials and Methods}

Medical records of 46 consecutive patients with a diagnosis of rhinocerebral mycosis admitted to the Neurology department from January 1997 to December 2005 were analyzed retrospectively. All the patients had histopathalogical evidence of fungal infection. Functional endoscopic excision biopsy using 0 degree and 30 degree endoscopes was done. Tissues were fixed in buffered formalin and processed for paraffin embedding. Hematoxylin and Eosin stained sections were examined for tissue reaction. Fungal morphology was identified by Periodic acid Schiff (PAS) and Gomori's methenamine silver (GMS) and other appropriate special stains. 'Broad non-septate hyaline ribbon-like hyphae with irregular branching' was the basis for identification as Zygomycetes and narrow hyphae with distinctive septa, parallel walls and acute angle dichotomous branching, for Aspergillus. Melanized fungi were identified by the presence of thin branching, septate hyphae with melanin pigment. The melanin-specific Masson-Fontana stain was used for confirmation. Culture reports were obtained whenever tissue was submitted for culture. The demographic data, predisposing illness, clinical features, radiological (MRI/CT) findings, treatment details (medical and/or surgical) and outcomes were recorded.

\section{Statistics}

Univariate analysis with odds ratio (OR) was employed in data analysis. Chi-square test was used for $P$ value and $P$ value of $<0.05$ was taken as significant.

\section{Results}

There were 46 patients with a diagnosis of rhinocerebral mycosis. Eight patients did not continue the treatment and were lost to follow-up. Hence the results were analyzed in 38 patients. Age ranged from 7-82 (mean 48.68) years; $30(79 \%)$ were males. All the patients presented with multiple symptoms and signs. Craniofacial pain was the most common initial presenting symptom, noted in 29 $(76.3 \%)$ patients. The other symptoms and signs were fever, vomiting, seizures, cranial nerve palsies, altered sensorium, meningitis and focal neurological deficits [Table 1]. At admission significant anemia (hemoglobin of $<8 \mathrm{gm} / \mathrm{dl})$ was noted in 15 (39.5\%), leukocytosis in $12(31.6 \%)$ and elevated erythrocyte sedimentation rate (ESR) in 22 (57.9\%) patients.

Rhinoorbital involvement with facial edema, proptosis, chemosis and ophthalmoplegia were the commonest findings noted in 26 (68.4\%) patients. Rhinoorbitocerebral
Table 1: Symptoms and signs of rhinocerebral mycosis at admission

\begin{tabular}{lc}
\hline Clinical feature & Number (\%) \\
Craniofacial pain & $29(76.3)$ \\
Fever & $14(37)$ \\
Vomiting & $7(18.4)$ \\
Seizures & $5(13.2)$ \\
Altered sensorium & $8(21)$ \\
Meningitis & $3(7.9)$ \\
Cranial nerve palsies & \\
$3^{\text {rd }} 4^{\text {th }}, 6^{\text {th }}$ cranial nerves & $36(94.7)$ \\
$2^{\text {nd }}$ cranial nerve & $30(78.9)$ \\
$5^{\text {th }}$ cranial nerve & $11(28.9)$ \\
$7^{\text {th }}$ cranial nerve & $6(15.8)$ \\
$8^{\text {th }}$ cranial nerve & $1(2.6)$ \\
$9^{\text {th }}$ and $10^{\text {th }}$ & $2(5.3)$ \\
$11^{\text {th }}$ and $12^{\text {th }}$ & $1(2.6)$ \\
Focal neurological deficits & $12(31.6)$ \\
\hline
\end{tabular}

involvement with focal neurological deficits was seen in $10(31.6 \%)$ patients. Sinocranial involvement with focal neurological deficits due to brain abscess was noted in two patients with mycosis due to melanized fungi. The third, fourth and sixth cranial nerves were involved in all the patients with rhinoorbital involvement and optic nerve in $30(79 \%)$ [Table 1] patients. Two patients had bilateral rhinoorbital involvement.

Immunocompromised state was noted in 24 (63.2\%); 23 had diabetes mellitus (DM) and one patient of Wegener's granulomatosis was on immunosuppressive therapy. One patient with DM had renal transplantation with hepatitis $C$ and $B$ virus infection and one patient with DM had HIV-1 infection. Seven patients with DM were in diabetic ketoacidosis (DKA), 12 had hyperglycemia and four were euglycemic at the time of admission. One patient had denovo DM with DKA. There were 14 (36.8\%) patients with no identifiable cause for immunosuppression.

Magnetic resonance imaging (MRI) was done in all the patients. Paranasal sinusitis with orbital involvement was noted in $26(68.4 \%)$ patients. Intracranial extension was noted in $12(31.6 \%)$ patients. Cavernous sinus involvement was noted in six and suprasellar extension in two patients. Stroke secondary to probable vasculitis was seen in 12 patients - 11 had internal carotid artery (ICA) stroke (bilateral involvement in two) and one had posterior cerebral artery (PCA) stroke. Four patients had intracranial abscess [Figure 1] (bifrontal -one, temporal lobe-one, capsuloganglionic -one and cerebellopontine angle-one). All the patients underwent sphenoethmoidectomy and or maxillectomy according to the involvement. Radical surgery (enucleation of eye, orbital exenteration, craniotomy with debridement, extensive craniofacial resection and reconstruction,) was done in $18(47.4 \%)$ patients.

The diagnosis of fungal infection was based on direct microscopy of the tissue from the nasal/sinus mucosa or the intracranial mass lesion and histopathology. Zygomycetes and Aspergillus were the commonest 


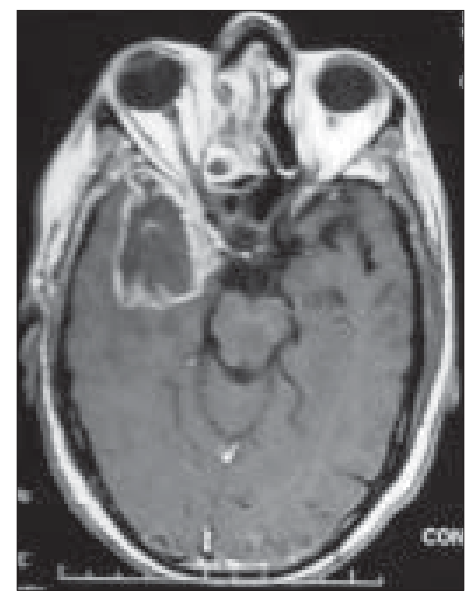

Figure 1a: MRI brain (contrast) image of a case of rhinocerebral mixed mucormycosis and Aspergillus infection with right temporal abscess (before treatment)
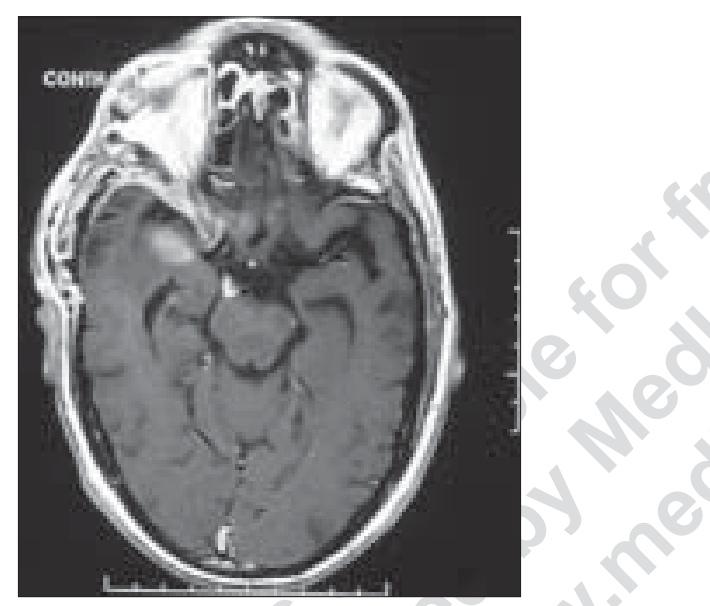

Figure 1b: MRI brain (contrast) image of a case of rhinocerebral mixed mucormycosis and Aspergillus infection with right temporal abscess (one year after treatment)

organisms found in $18(47.4 \%)$ and 16 (42.1\%), respectively [Figures 2 and 3]. Melanized fungi was found in two and mixed Aspergillus and Zygomycosis in two patients. The culture was done in 21 cases and it was positive in 12 (aspergillus species in five, rhizopus in six and cladosporium in one). Aspergillus flavus was isolated in three, Aspergillus fumigatus and Aspergillus terreus in one each. Among those with culture-positive zygomycosis, Rhizopus oryzae was isolated in five and rhizomucor in one patient. Culture was positive in one patient with pheohyphomycosis and basal ganglionic abscess and cladosporium species was isolated.

All these patients were treated with amphotericin B. In addition six patients with Aspergillosis received 5 -flurocytosine. The total dose of amphotericin B given ranged from 3-6 g. Liposomal amphotericin (Ambisome) was given to seven patients and the total dose given in each patient was $3 \mathrm{~g}$. One patient with rhinoorbitocerebral mixed Aspergillus and Zygomycosis

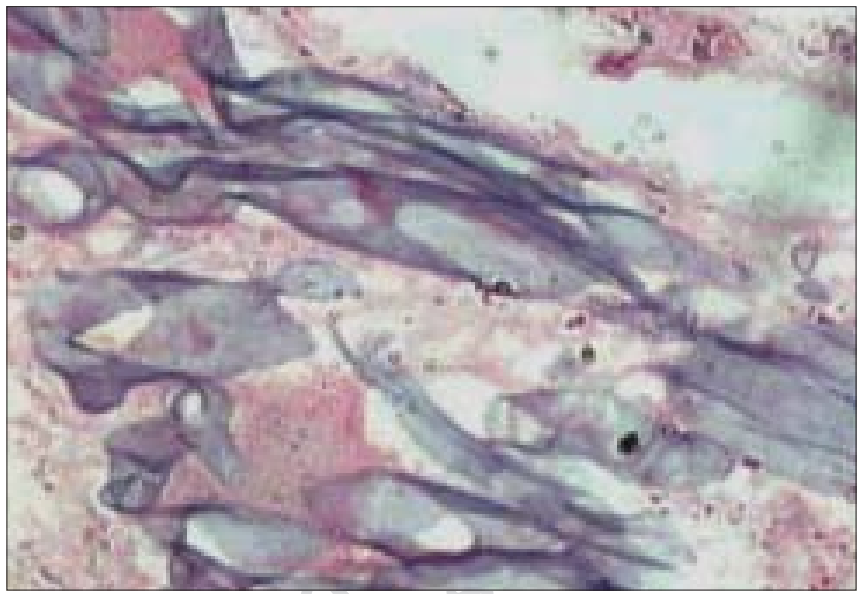

Figure 2: Photomicrograph showing broad, aseptate irregularly branching hyphae of mucormycosis (H/E, x40)

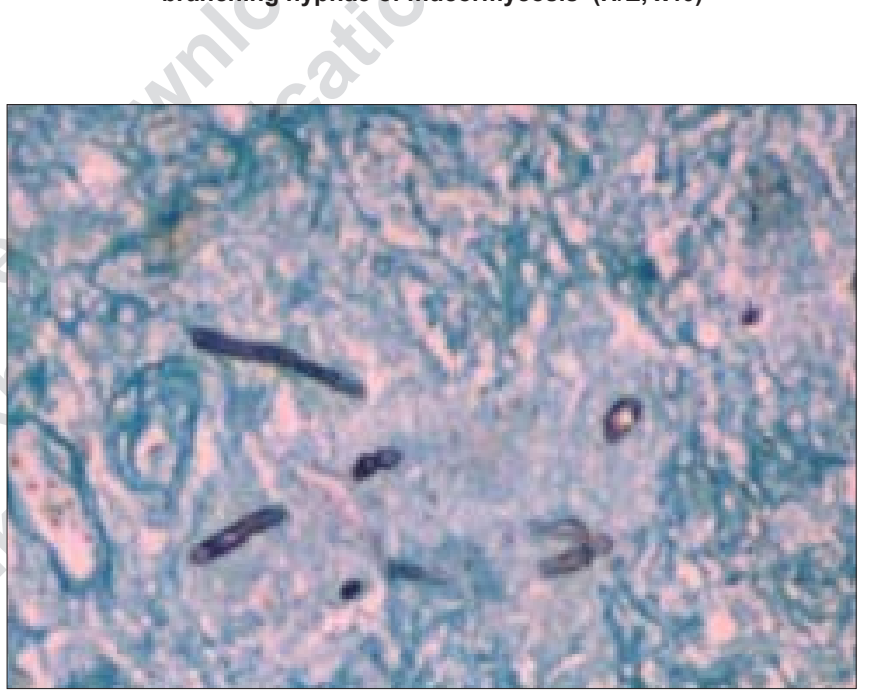

Figure 3: Photomicrograph showing slender, septate hyphae of Aspergillus species (Gomori's methenamine sliver, $\mathbf{x 4 0}$ )

infection and a temporal lobe fungal abscess, did not respond to liposomal amphotericin. Mixed zygomycosis and aspergillus infection was identified on tissue morphology, but culture isolated Aspergillus fumigatus only. He was given intravenous voriconazole $400 \mathrm{mg} /$ day for two weeks followed by oral voriconazole $400 \mathrm{mg} /$ day for six months. The patient improved markedly at the end of one year with no residual neurological deficits and near total resolution of the temporal lobe abscess on MRI [Figure 1]. The common complications during treatment with amphotericin B were hypokalemia in 30 (78.9\%), acute renal failure in four (10.5\%), hyponatremia in two (5.3\%) and symptomatic hypomagnesemia in three (7.9\%). The duration of hospital stay in the survived group was 21-123 (mean 56) days.

All the patients underwent follow-up MRI of brain and sinuses (plain and contrast) for the resolution of the lesions. Repeat biopsy/aspiration cytology was done in 12 patients who survived and no fungal elements could be demonstrated on pathology. 
The follow-up ranged from one to nine years. Eighteen (47.4\%) patients died. Elderly patients (age $>60$ years) had a mortality of $58.3 \%$ compared to $36.4 \%$ in adults (age 17-59) and the difference was not statistically significant $(\mathrm{P} \leq 1)$. There was no statistically significant difference in mortality between males (46.6\%) and females (50\%) with $\mathrm{P} \leq 1$. Patients with focal neurological deficits had higher mortality (75\%) compared to those without focal deficits (34.6\%) and the difference between the two groups is statistically significant $(P<0.025)$. The mortality was higher in patients with immunocompromised state (56.5\%) compared to those with immunocompetent state (33.3\%) but the difference was not statistically significant $(\mathrm{P} \leq$ 0.2 ). No significant difference in mortality was noted between the patients who underwent radical surgery $(44.4 \%)$ and no radical surgery (50\%), $P \leq 1$. Among the 10 patients who did not undergo radical surgery six patients died due to associated medical complications like septicemia in three, massive upper gastrointestinal bleed in one, acute renal failure in one and myocardial infarction in one. One patient had subarachnoid hemorrhage before death. The mortality was higher in rhinocerebral syndrome due to Zygomycosis (62.5\%) than with Aspergillus (38.9\%), but the difference was not statistically significant $(P \leq 0.2)$. The mortality was $66.6 \%$ in patients with significant anemia at admission and $38.5 \%$ in those without anemia, the difference was not significant $(\mathrm{P} \leq 1)$. The predictors for mortality: odds ratio (95\% CI) were 2.45 (1.01-3.89) for elderly age, 5.67 (4.137.21) for intracranial extension with focal neurological deficit, 2.6 (1.26-3.94) for immunocompromised state, 2.62 (1.25-3.99) for infection with zygomycosis and 2.33 (1.01-3.65) for anemia [Table 2]. The OR was highest for intracranial extension causing focal neurological deficit,

\begin{tabular}{|c|c|c|}
\hline \multicolumn{3}{|c|}{ Table 2: Predictors of mortality in rhinocerebral mycosis } \\
\hline Variable & Mortality \% & Odds ratio $(95 \% \mathrm{Cl})$ \\
\hline \multicolumn{3}{|l|}{ Age } \\
\hline Elderly vs. & 58.3 & $2.45(1.01-3.89)$ \\
\hline Adults & 36.4 & \\
\hline \multicolumn{3}{|l|}{ Sex } \\
\hline Males vs. & 46.7 & $0.875(0.68-2.43)$ \\
\hline Females & 50 & \\
\hline \multicolumn{3}{|c|}{ Focal neurological deficits } \\
\hline Present vs. & 75 & $5.67(4.13-7.21)$ \\
\hline Absent & 34.6 & \\
\hline \multicolumn{3}{|c|}{ Immunocompromised state } \\
\hline Present vs. & 56.5 & $2.6(1.26-3.94)$ \\
\hline Absent & 33.3 & \\
\hline \multicolumn{3}{|l|}{ Anemia } \\
\hline Present vs. & 60 & $2.33(1.01-3.65)$ \\
\hline Absent & 39.1 & \\
\hline \multicolumn{3}{|l|}{ Radical surgery } \\
\hline Done vs. & 44.4 & $0.8(0.5-2.1)$ \\
\hline Not done & 50 & \\
\hline \multicolumn{3}{|l|}{ Type of infection } \\
\hline Zygomycosis vs. & 62.5 & $2.62(1.25-3.99)$ \\
\hline Aspergillus & 38.9 & \\
\hline
\end{tabular}

suggesting that it is a major predictor of mortality in rhinocerebral mycosis.

\section{Discussion}

Rhinocerebral fungal infection typically originates in the nasal or oral mucosae, spreads to paranasal sinuses and enters the orbit via the ethmoid and maxillary sinuses or via the nasolacrimal duct. Intracerebral extension may occur from the orbit via the orbital apex, orbital vessels or via the cribriform plate. Diabetes mellitus is a common predisposing factor for this infection. ${ }^{[4,6-10,12,17,18]}$ CNS mycosis is common in the setting of diabetic ketoacidosis (DKA). In our series diabetes was noted in 23 (56.5\%) patients and seven were in DKA at admission. However, rhinoorbital zygomycosis has been reported without ketoacidosis and with well-controlled diabetes ${ }^{[19]}$ Euglycemic state at admission was noted in four patients in our series. Postrenal transplant state and retrovirus (HIV-1) infection were the other predisposing factors similar to previous reports. ${ }^{[8,20,21]}$ Occurrence in immunologically competent patients is not uncommon ${ }^{[5,22,23]}$ and we had 14 (36.8\%) patients with immunocompetent state.

Ocular and sinonasal involvement were the commonest clinical features at admission..$^{[6,8-10,12]}$

In our series rhinoorbital involvement was noted in 26 patients and 12 had rhinoorbitocerebral involvement on imaging. Cavernous sinus invasion with ICA thrombosis is a common complication, associated with high mortality. ${ }^{[24-28]}$ In the present series six patients had cavernous sinus involvement on MR imaging and two had suprasellar extension. Eleven patients had ICA stroke and one had PCA stroke, probably due to fungal vasculitis. Intracerebral abscess is another complication of invasive mycosis, especially in intravenous drug users. ${ }^{[29-31]}$ We had four patients with intracranial abscess. In two of these patients melanized fungi were identified. Fatal complications like acute subdural hematoma and intracerebral hemorrhage due to rupture of an aneurysm was reported. ${ }^{[32]}$ In the present study one patient developed subarachnoid hemorrhage during treatment and died.

Invasive diseases caused by fungi are termed mycoses. Aspergillus is the most common fungus in histologically verified CNS mycosis from India and presents with focal neurological signs and symptoms. ${ }^{[33-36]}$ Zygomycosis is infrequently reported from India and the rhinocerebral form is the commonest form of CNS Zygomycosis. ${ }^{[37]}$ Aspergillus spreads to the CNS by direct inoculation by trauma or surgery, direct extension from paranasal sinuses or eye, invasion of arteries and veins and fungemia. The inflammatory response may range from an acute exudative lesion to a calcified granulomatous lesion. Zygomycosis is caused by direct extension from paranasal sinuses. Characteristic morphology of the 
fungus on histopathology invading the vessel wall with fibrin deposition, intense polymorphonuclear response and infarction of adjacent tissue help in diagnosis. Multiple melanized fungi also cause central nervous system fungal infections. Cladosporium bantiana is the most commonly isolated species among phaeohyphomycosis, the dark pigmented fungi. It occurs most commonly in immunocompetent patients and brain abscess is the commonest presentation. The mortality was high (70\%) regardless of immune status. ${ }^{[38]}$ We identified Cladosporium species in one case and in the second a tissue diagnosis of melanized fungus was made. Sinocranial disease, angioinvasion on histology has been reported with Cladosporium. ${ }^{[23]}$ Fungus-specific virulent factors, such as expression of surface molecules allowing adhesion to endothelial and epithelial cells, the production of surface molecules mimicking host substances and thereby avoiding the host's immune response and hydrolytic enzymes are important in the pathogenesis. ${ }^{[39]}$ Biopsy/aspiration cytology will confirm the diagnosis and must be performed in all suspected cases. Aspergillus and zygomycosis are the commonest organisms causing rhinocerebral syndrome..$^{[5-8,12,18]}$ In the present study Aspergillus was found in $47.4 \%$ and Zygomycosis in $42.1 \%$. Melanized fungi was found in two and mixed Aspergillus and mucor in two patients. Mixed infection with Aspergillus and Zygomycosis has been reported earlier. ${ }^{[5,40]}$

Contrast MRI of the brain and sinuses should be done in all patients with suspected rhinocerebral mycosis. Contrast enhancement, extension of the disease into the orbit and cranial cavity can be well delineated on MRI and may simulate malignancy. ${ }^{[5]}$ Aggressive therapy with amphotericin B, rigorous glucose control, surgical debridement and radical surgery when indicated will improve the survival in these patients. ${ }^{[7-10,12,13,15,24,25,29,40]}$ Radical surgery was performed in addition to amphotericin B therapy in $47.4 \%$ of patients in the present study. Prolonged therapy with amphotericin may be needed in some cases. Voriconazole may be tried in resistant cases of rhinocerebral aspergillosis. ${ }^{[41]}$ Only one patient with mixed Zygomycosis and Aspergillus infection received voriconazole in the present series and responded well. Mixed zygomycosis and aspergillus infection was identified on tissue morphology, but culture isolated Aspergillus fumigatus only. Several times Aspergillus may be misdiagnosed as zygomycetes on tissue morphology. The role of voriconazole in rhinocerebral mycosis needs further confirmation in a large series of patients.

Mortality is high in patients with rhinocerebral mycosis if treatment is delayed. ${ }^{[7-9,35]}$ Intracranial extensions of the disease on imaging, hemiplegia as a neurological complication and diabetic ketoacidosis at presentation are adverse prognostic factors. ${ }^{[11,12,42]}$ The predictors of mortality in the present series were elderly age, intracranial extension, immunocompromised state, infection with Zygomycosis and anemia. Intracranial extension causing focal neurological deficit is a major predictor of mortality. Awareness, early diagnosis, aggressive surgical and medical management with antifungal therapy and treatment of underlying predisposing factors will improve the prognosis.

One of the limitations of the present study was that the fungus was identified on tissue sections based on morphology of fungi. Aspergillus, Fusarium, Scedosporium, Pseudallescheria species and Chaetomium species exhibit similar septate tissue hyphae in tissue sections. Correlation with culture results is necessary for definite identification of the species. ${ }^{[43,44]}$

\section{Conclusion}

Intracranial extension causing focal neurological deficit is a major predictor of mortality in rhinocerebral mycosis. Aggressive medical management with prompt surgical therapy is an important factor contributing to a better outcome.

\section{References}

1. Paltauf A. Mycosis mucorina. Virchows Arch 1885;102:543-64.

2. Eucker J, Sezer O, Graf B, Possinger K. Mucormycosis. Mycoses 2001;44:253-60.

DeShazo RD, O'Brien M, Chapin K, Soto-Aguilar M, Gardner L, Swain R. A new classification and diagnostic criteria for invasive fungal sinusitis. Arch Otolaryngol Head Neck Surg 1997;123:1181-8.

4. Yohai RA, Bullack JD, Aziz AA, Markert RJ. Survival factors in Rhinoorbitocerebral mucormycosis. Surv Ophthalmol 1994;39:3-22.

5. Hussain S, Salahuddin N, Ahmad I, Salahuddin I, Hooma R. Rhinocerebral invasive mycosis: Occurrence in immunocompetent individuals. Eur J Radiol 1995;20:151-5.

6. Hosseini SM, Borghei P. Rhinocerebral mucormycosis: Pathways of spread. Eur Arch Otorhinolaryngol 2005;262:932-8.

7. Safar A, Marsan J, Margiani O, Al-Sebeih K, Al-Harbi J, Valvoda M. Early identification of rhinocerebral mucormycosis. J Otolaryngol 2005;34:166-71

8. Dubey A, Patwardhan RV, Sampath S, Santosh V, Kolluri S, Nanda A. Intracranial fungal granuloma: Analysis of 40 patients and review of the literature. Surg Neurol 2005;63:254-60.

9. Khor BS, Lee MH, Leu HS, Liu JW. Rhinocerebral mucormyocosis in Taiwan. J Microbiol Immunol Infect 2003;36:266-9.

10. Rangel-Guerra RA, Martinez HR, Saenz C, Bosques-Padilla F, EstradaBellmann I. Rhinocerebral and systemic mucormycosis. Clinical experience with 36 cases. J Neurol Sci 1996;143:19-30.

11. Butugan O, Sanchez TG, Goncalez F, Venosa AR, Miniti A. Rhinocerebral mucormyocisis: Predisposing factors, diagnosis, therapy, complications and survival. Rev Laryngol Otol Rhinol (Bord) 1996;117:53-5.

12. Nussbaum ES, Hall WA. Rhinocerebral mucormycosis: Changing patterns of disease. Surg Neurol 1994;41:152-6.

13. Chetchotisakd P, Boonma P, Sookpranee M, Pairojkul C. Rhinocerebral mucormycosis: A report of eleven eases. Southeast Asian J Trop Med Public Health 1991;22:268-73.

14. Ochi JW, Harris JP, Feldman JI, Press GA. Rhinocerebral Mucormycosis: Results of aggressive surgical debridement and amphotericin B. Laryngoscope 1988;98:1339-42.

15. Pillsbury HC, Fischer ND. Rhinocerebral mucormycosis. Arch Otolaryngol 1977;103:600-4

16. Petrikkos G, Skiada A, Sambatakou H, Toskas A, Vaipoulos G, 
Giannopoulou M, et al. Mucormycosis: Ten year experience at a tertiary care center in Greece. Eur J Clin Microbiol Infect Dis 2003;22:753-6.

17. Ferry AP, Abedi S. Diagnosis and management of rhino-orbitocerebral mucormycosis (phycomycosis). A report of 16 personally observed cases. Ophthalmology 1983;90:1096-104.

18. Sundaram C, Mahadevan A, Laxmi V, Yasha TC, Santosh V, Murthy JM, et al. Cerebral zygomycosis. Mycoses 2005;48:396-407.

19. Ellis CJ, Daniel SE, Kennedy PG, Oppenheimer SM, Scaravilli F. Rhinoorbital zygomycosis. J Neurol Neurosurg Psychiatary 1985;48:455-8.

20. Morduchowicz G, Shmucli D, Shapira Z, Cohen SL, Yussim A, Block CS, et al. Rhinocerebral mucormycosis in renal transplant recipients: Report of three cases and review of the literature. Rev Infect Dis 1986;8:4416.

21. Moraru RA, Grossman ME. Palatal necrosis in an AIDS patient: A case of mucormycosis. Cutis 2000;66:15-8.

22. Kim DG, Hong SC, Kim HJ, Chi JG, Han MH, Choi KS, et al. Cerebral aspergillosis in immunologically competent patients. Surg Neurol 1993;40:326-31.

23. Sundaram C, Umabala P, Laxmi V, Purohit AK, Prasad VS, Panigrahi M, et al. Pathology of fungal infections of the central nervous system: 17 years' experience from Southern India. Histopathology 2006:49:396405 .

24. Simmons JH, Zeitler PS, Fenton LZ, Abzug MJ, Fiallo-Scharer RV, Klingensmith GJ. Rhinocerebral mucormycosis complicated by internal carotid artery thrombosis in a pediatric patient with type I diabetes mellitus: A case report and review of the literature. Pediatr Diabetes 2005;6:234-8.

25. Delbrouck C, Jacobs F, Fernandez Aguilar S, Devroede B, Choufani G, Hassid S. Carotid artery occlusion due to fulminant rhinocerebral mucormycosis. Acta Otorhinolaryngol Belg 2004;58:135-40.

26. Anaissie E., Shikhani AH. Rhinocerebral mucormycosis with internal carotid occlusion: Report of two cases and review of the literature. Laryngoscope 1985;95:1107-13.

27. Galetta SL, Wulc AE, Goldberg HI, Nichols CW, Glaser JS. Rhinocerebral mucormycosis: Management and survival after carotid occlusion. Ann Neurol 1990;28:103-7.

28. Quattrocolo G, Pignatta P, Dimanico U, Tarenzi L, Bagglore P. Rhinocerebral mucormycosis and internal carotid artery thrombosis in a previously healthy patient. Acta Neurol Belg 1990;90:20-6.

29. Pellacchia V, Terenzi V, Moricca LM, Buonaccorsi S, Indrizzi E, Fini G. Brain abscess by mycotic and bacterial infection in a diabetic patient: Clinical report and review of literature. J Craniofac Surg 2006;17:57884.

30. Fong KM, Seneviratne EM, McCormack JG. Mucor cerebral abscess associated with intravenous drug abuse. Aust N Z J Med 1990;20:74-7.

31. Stave GM, Heimberger T, Kerkering TM. Zygomycosis of the basal ganglia in intravenous drug users. Am J Med 1989;86:115-7.

32. Ho KL. Acute subdural hematoma and intracerebral hemorrhage. Rare complications of rhinocerebral mucormycosis. Arch Otolaryngol $1979 ; 105: 279-81$

33. Santosh V, Yasha TC, Khanna N. Fungal infections of the nervous system: A pathological study. Neurol Infect Epidemiol 1996;1:69-79.

34. Sharma BS, Khosla VK, Kak VK, Banerjee AK, Vasishtha RK, Prasad KS, et al. Intracranial fungal granuloma. Surg Neurol 1997;47:48997 .

35. Murthy JM, Sundaram C, Prasad VS, Purohit AK, Rammurthi S, Laxmi V. Sinocranial aspergillosis a form of central nervous system aspergillosis in South India. Mycoses 2001;44:141-5.

36. Murthy JM, Sundaram C, Prasad VS, Purohit AK, Rammurthi S, Laxmi V. Aspergillosis of central nervous system: A study of 21 patients seen in a university hospital in South India. J Assoc Physicians India 2000;48:677-81.

37. Chakrabarti A, Das A, Mandal J, Shivaprakash MR, George VK, Tarai B, et al. The rising trend of invasive zygomycosis in patients with uncontrolled diabetes mellitus. Med Mycol 2006;44:335-42.

38. Revankar SG, Sutton DA, Rinaldi MG. Primary central nervous system phaeohyphomycosis: A review of 101 cases. Clin Infect Dis 2004;38:20616.

39. Levitz SM. Overview of host defenses in fungal infections. Clin Infect Dis $1992 ; 14: S 37-42$.

40. Alfano C, Chiummariello S, Dessy LA, Bistoni G, Scuderi N. Combined mucormycosis and Aspergillosis of the rhinocerebral region. In Vivo 2006;20:311-5

41. Elter T, Sieniawski M, Gossmann A, Wickenhauser C, Schröder U, Seifert H, et al. Voriconazole brain tissue levels in rhinocerebral aspergillosis in a successfully treated young woman. Int J Anitimicrob Agents 2006;28:262-5.

42. Dhiwakar M, Thakar A, Bahadur S. Improving outcomes in rhinocerebral mucormycosis-early diagnostic pointers and prognostic factors. J Laryngol Otol 2003;117:861-5.

43. Kleinschmidt-DcMasters BK. Central nervous system. Aspergillosis: A 20 year retrospective series. Hum Pathol 2002:33:116-24.

44. Tarrand JJ, Lichterfeld M, Warraich I, Luna M, Han XY, May GS, et al. Diagnosis of invasive septate mold infections. A correlation of microbiological culture and histologic or cytologic examination. Am J Clin Pathol 2003:119;854-8.

Accepted on 04-07-2007

Source of Support: Nil, Conflict of Interest: None declared. 